\title{
Preoperative Analysis of Optimal Imaging Orientation in Fluoroscopy for Voxel-Based 2-D/3-D Registration
}

\author{
Yoshikazu Nakajima ${ }^{1}$, Yuichi Tamura ${ }^{2}$, Yoshinobu Sato ${ }^{1}$, \\ Takahito Tashiro ${ }^{1}$, Nobuhiko Sugano ${ }^{3}$, Kazuo Yonenobu ${ }^{4}$, Hideki Yoshikawa ${ }^{3}$, \\ Takahiro Ochi ${ }^{2}$, and Shinichi Tamura ${ }^{1}$ \\ Division of Interdisciplinary Image Analysis ${ }^{1}$ \\ Division of Computer Integrated Orthopaedic Surgery ${ }^{2}$, \\ Department of Orthopaedic Surgery ${ }^{3}$, \\ Osaka University Graduate School of Medicine, Suita, Osaka 565-0871, Japan \\ Osaka Minami National Hospital ${ }^{4}$, Kawachinagano, Osaka 586-8521, Japan
}

\begin{abstract}
We have developed a system for the 3-D localization of anatomical structures without the need for surgical exposure by using multiple-view fluoroscopy images. In this paper, we describe the system and evaluate its application to the estimation of optimal imaging orientations in fluoroscopy. For positional measurement, a voxel-based 2-D/3-D registration technique was employed. Since the measurement condition depends on the object shape, spatial distribution of X-ray absorption, and overlap of organs or structures (which differs at each imaging position), determining the optimal combination of fluoroscopy orientations is significant. We propose a system for preoperative determination of the optimal imaging orentation by using the accuracy estimation of stereo localization from single-plane localization results. In an experiment, the computation time needed was 10 hours, which was about 14 times shorter than the time required for a full search of imaging orientation combinations.
\end{abstract}

\section{Introduction}

Two-dimensional (2-D)/three-dimensional (3-D) registration between an intraoperative fluoroscopy image and a preoperative 3-D CT image [1] 2] is an effective means of organ localization without surgical exposure. Primary research in 2-D/3-D registration for medical applications was based on the contour-surface matching technique [3] 4] [5]. The contour-based method is, however, not stable with respect to false contours, and exact extraction of bone edges is not always feasible because of material heterogeneity and overlap of peripheral organs. On the other hand, voxel-based registration methods, which use digitally reconstructed radiographs (DRRs) generated from a 3-D CT image, are generally robust compared with the contour-based method [6] [7] 8] 9] [10]. For these reason, we have employed a voxel-based method [6] for our purpose of vertebra localization. 
For accurate pose measurement, registration from two stereo images has been proposed [3] 11. The object shape, distribution of X-ray absorption, and overlap of peripheral organs or structures — which changes along with the imaging orientation - cause spatial heterogeneity of the pose measurement accuracy. Therefore, to improve the localization accuracy, it is significant to optimize the imaging orientations. Some approaches to this have been reported [12] [13] [14] [15], but, optimal view analysis for registration has not hitherto been proposed. Here, we discuss determination of the optimal imaging orientation in fluoroscopy for voxel-based 2-D/3-D registration.

\section{Methods}

\subsection{Voxel-Based 2-D/3-D Registration}

The algorithm for voxel-based 2-D/3-D registration [6] 7] consists of three major components: 2-D image (DRR) generation from a 3-D CT image, similarity evaluation between a DRR and a fluoroscopy image, and optimization. In the DRR generation process, segmentation of the anatomical structure of interest in the pre-operative 3-D CT image and pseudo projections to generate a DRR (Fig. 1 (a)) from the segmented CT volume are performed. In the similarity evaluation, the gradient correlation is employed for single-plane pose measurement, which is given by

$$
\begin{aligned}
& G C=\frac{1}{2} \frac{\sum_{(i, j) \in T_{\partial_{i}}} F_{i} D_{i}}{\sqrt{\sum_{(i, j) \in T_{\partial_{i}}} F_{i}^{2}} \sqrt{\sum_{(i, j) \in T_{\partial_{i}}} D_{i}^{2}}}+\frac{1}{2} \frac{\sum_{(i, j) \in T_{\partial_{j}}} F_{j} D_{j}}{\sqrt{\sum_{(i, j) \in T_{\partial_{j}}} F_{j}^{2}} \sqrt{\sum_{(i, j) \in T_{\partial_{j}}} D_{j}^{2}}}, \\
& \text { where } \quad F_{i}=\frac{\partial I_{f l}(i, j)}{\partial i}-\frac{\overline{\partial I_{f l}}}{\partial i}, \quad D_{i}=\frac{\partial I_{D R R}(i, j)}{\partial i}-\frac{\overline{\partial I_{D R R}}}{\partial i}, \\
& \text { and } \quad F_{j}=\frac{\partial I_{f l}(i, j)}{\partial j}-\frac{\overline{\partial I_{f l}}}{\partial j}, \quad D_{j}=\frac{\partial I_{D R R}(i, j)}{\partial j}-\frac{\overline{\partial I_{D R R}}}{\partial j},
\end{aligned}
$$

and $I_{f l}$ and $I_{D R R}$ are the pixel intensities of the fluoroscopy image and DRR, respectively. $\partial I_{f l} / \partial i, \partial I_{f l} / \partial j, \partial I_{D R R} / \partial i$, and $\partial I_{D R R} / \partial j$ are the pixel intensities of horizontal and vertical gradient images of the fluoroscopy image and DRR, respectively. For stereo imaging measurement, the evaluation function — which is the sum of the gradient correlations of both imaging positions - is used. Estimation of the CT volume pose is realized by an optimization approach based on the Powell method [6] [7.

In our experiments, an anatomical coordinate system of the vertebra of interest (Fig. 2 (b)) [16] is determined by manually specifiying surface points on the backface and topface (Fig. 2 (a)) in the 3-D CT image.

\subsection{Estimation of Optimal Imaging Orientation}

We propose a method of estimating the optimal imaging orientation in fluoroscopy for stereo $2-\mathrm{D} / 3-\mathrm{D}$ registration. Since the cost of error computation for 2-D/3-D registration is relatively high, a method of estimating the stereo localization accuracy from single-plane localization results is employed. An overview of the system is shown in Fig. 3] From a CT volume and position parameters, an 


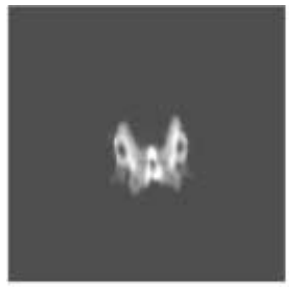

(a)

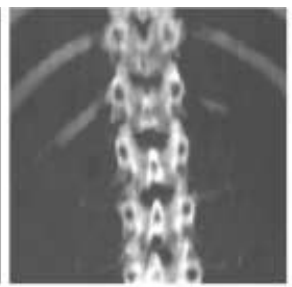

(b)

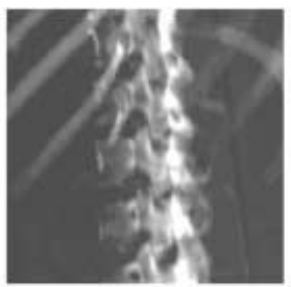

(c)

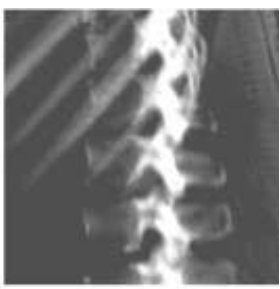

(d)

Fig. 1. Digitally reconstructed radiographs (DRRs). (a) DRR from segmented CT image of L1 vertebra. (b),(c),(d) DRR from original CT image. The imaging angles are $0 \mathrm{deg}, 45 \mathrm{deg}$, and $90 \mathrm{deg}$, respectively. Overlaps of vertebrae, ribs and soft tissues change along with the imaging angle.

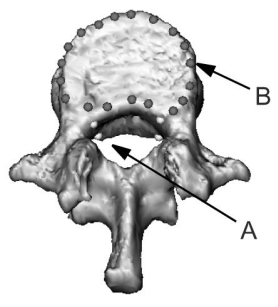

(a)

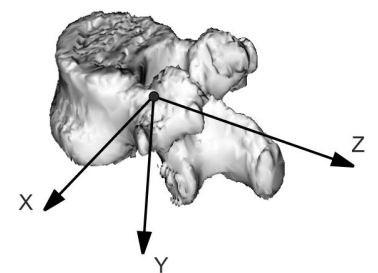

(b)

Fig. 2. Anatomical coordinate system of a vertebra. (a) Sampling points on the vertebra surface. A (set of white four points) determines the origin and the backface. B (set of gray points) determines the topface. (b) Anatomical coordinate system determined by the origin, backface, and topface.

X-ray fluoroscopy image is generated for the computer simulation. The CT volume of the vertebra of interest is segmented in the original CT volume. Initial estimates of the position parameters, which are $\left(t_{x}, t_{y}, t_{z}\right)$ for translation and $\left(\theta_{x}, \theta_{y}, \theta_{z}\right)$ for rotation, are made by adding random noise to the true position parameters. Then, 2-D/3-D registration is performed. By changing the position parameters of the $\mathrm{CT}$ volume from the imaging system, an error profile of singleplane localization is computed. Errors of translation and rotation are described as a covariance matrix, and the calculated covariance matrix is transformed in local coordinate system of the anatomical structure. The transformed matrix $\dot{C}_{\text {single_plane }}$ in the local coordinate system of the anatomical structure is given by

$$
\dot{C}_{\text {single_plane }}=\left(M^{T} M\right)^{-1} M^{T} C_{\text {single_plane }}\left(\left(M^{T} M\right)^{-1} M^{T}\right)^{T},
$$

where $C_{\text {single_plane }}$ is the covariance matrix of rotation and translation errors, and $M$ is the Jacobian of transformation between the local coordinate system of target anatomical structure and the coordinate system of the imaging plane. 
The error of stereo localization, determined by using a combination method of error distribution [17], is given by

$$
C_{\text {stereo }}=\dot{C}_{\text {single_plane } 2}\left(\dot{C}_{\text {single_plane } 1}+\dot{C}_{\text {single_plane } 2}\right)^{-1} \dot{C}_{\text {single_plane } 1},
$$

where $C_{\text {stereo }}$ is the covariance matrix of stereo measurement and $C_{\text {single_plane } 1}$ and $C_{\text {single_plane } 2}$ are covariance matrices of single-plane measurement. Then, to project the error into the local coordinate axes of the anatomical structure for clinical evaluation, it is approximately evaluated by fitting a 3-D Gaussian function $G\left(x, y, z ; \sigma_{x}, \sigma_{y}, \sigma_{z}\right)$ the axes of which correspond to the local coordinate system of the anatomical structure.

When the number of imaging orientations for the optimal orientation analysis of single-plane localization is $n$, the combination number of imaging orientations for the optimal orientation analysis of stereo localization is $\frac{1}{2} n(n+1)$. Since the proposed method only requires the accuracy of single-plane localization, the number of imaging orientations for accuracy analysis is $n$ and the computation time of an iteration is half that required for stereo localization. In the case of 15-degree resolution (13 positions) of imaging orientation and 50 iterations at each orientation, the computation time needed for the proposed method was 10 hours (with a Pentium Xeon $1.7 \mathrm{GHz}, 2 \mathrm{CPUs}$, and $2 \mathrm{~GB}$ memory) which is $1 / 14$ that required for a full search in imaging orientation pairs.

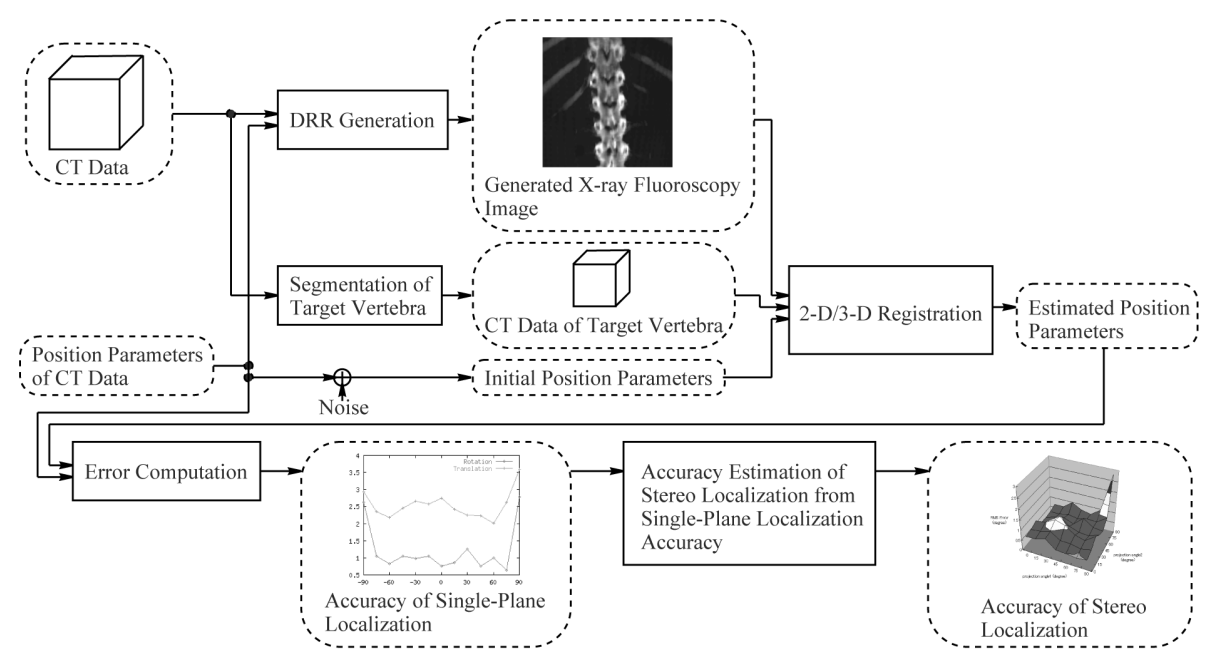

Fig. 3. Accuracy estimation of stereo localization

\section{Experiment}

\subsection{Effects of Overlap}

We assessed the effects of organ or other structure overlap. Since our 2-D/3-D registration method registers a segmented $\mathrm{CT}$ volume of the vertebra of interest, 
overlap of upper and lower vertebrae, ribs, and soft tissues causes pose estimation error. The CT data used in the experiment sections were a set of abdominal images that were imaged for clinical diagnosis of intestinal disease. The FOV, slice thickness, and matrix size were $360 \mathrm{~mm}, 2.0 \mathrm{~mm}$, and $512 \times 512 \times 128$ pixels, respectively. The images included the lower thoracic and lumber spine (from T10 to $\mathrm{C} 4$ ). In the system, the CT volume was segmented to parts of the vertebra of interest (L1 vertebra), other vertebrae, ribs, and soft tissues. By controlling of their visibility, we evaluated the effect of organ and other structre overlap. The experimental conditions are shown in Table 1. In the experiment, the matrix size of generated 2 -D image was $128 \times 128$ pixels. Let $\sigma_{\text {error } 0}, \sigma_{\text {error } 1}$, and $\sigma_{\text {error } 2}$ be standard deviations of error in conditions 0,1 , and 2 , respectively, and $\sigma_{\text {effect } 0}$, $\sigma_{\text {effect } 1}$, and $\sigma_{\text {effect } 2}$ the overlap effect of each organ or structure, respectively. The overlap effects are given by

$$
\begin{aligned}
& \sigma_{\text {effect } 0}=\sigma_{\text {error } 0}, \\
& \sigma_{\text {effect } 1}=\sqrt{\sigma_{\text {error } 1}^{2}-\sigma_{\text {error } 0}{ }^{2}}, \\
& \text { and } \sigma_{\text {effect } 2}=\sqrt{\sigma_{\text {error } 2^{2}-\sigma_{\text {error }}{ }^{2}}} \text {, }
\end{aligned}
$$

respectively.

Table 1. Experimental Conditions for Error Analysis of Organ or Other Structure Overlap

\begin{tabular}{|l|c|c|c|}
\hline Segmented part & Condition 0 & Condition 1 & Condition 2 \\
\hline L1 vertebra & visible & visible & visible \\
\hline vertebrae & invisible & visible & visible \\
\hline ribs and soft tissues & invisible & invisible & visible \\
\hline
\end{tabular}

The results are shown in Fig. 4. The error of localization of the L1 vertebra was $0.43 \pm 0.45$ degree and $1.27 \pm 0.70 \mathrm{~mm}$. The error caused by the overlap of the upper and lower vertebrae was $0.76 \pm 0.29$ degree and $1.15 \pm 0.60 \mathrm{~mm}$. The error caused by the overlap of ribs and soft tissues was $0.01 \pm 0.11$ degree and $0.11 \pm 0.38 \mathrm{~mm}$, which was smaller than the other errors. In a clinical CT image for spine surgery, the imaging volume is limited to the area around the surgical site and does not include all of the ribs and soft tissues. The above results showed the feasibility of preoperative estimation of the optimal imaging orientation using a segmented CT image of vertebrae in clinical use.

\subsection{Accuracy Computation of Single-Plane Localization}

The localization accuracy of the single-plane measuremenst was validated. The results are shown in Fig. 5. In the figure, the optimal orientation was \pm 60 degrees on the $y$-axis rotation. On the $x$-axis rotation, tilting of less than \pm 15 degrees might be acceptable. The results for over \pm 15 degrees of tilt were affected by the overlap of upper/lower vertebrae. 


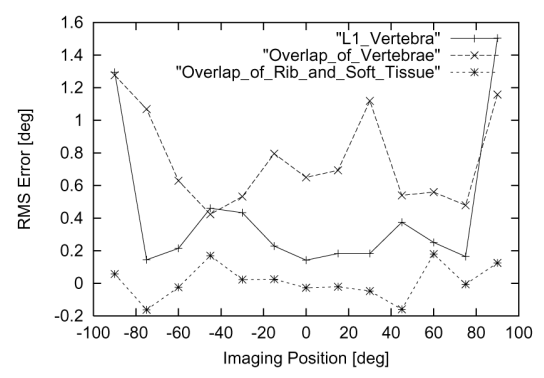

(a)

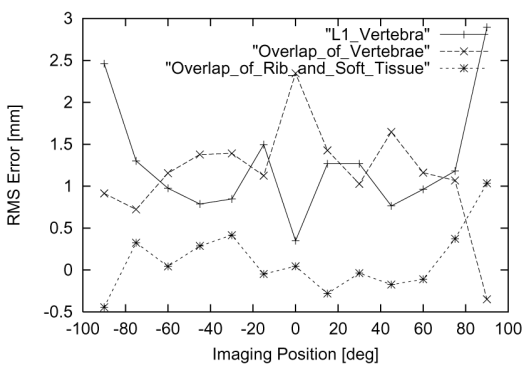

(b)

Fig. 4. Effects of tissue overlap. (a) Rotation error. (b) Translation error.

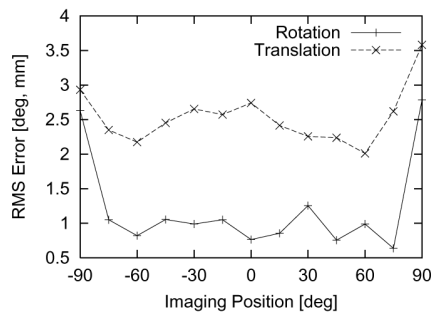

(a)

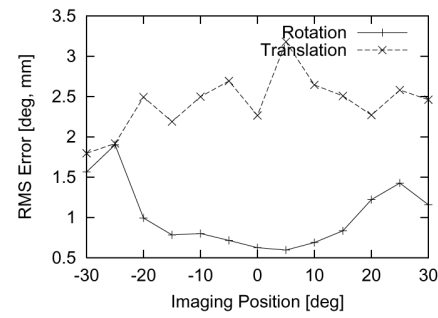

(b)

Fig. 5. Localization accuracy of single-plane measurement. The horizontal axis shows the imaging angle of the fluoroscope in the vertebra coordinate system. The vertical axis shows the root mean square (RMS) errors of the localized pose of the target vertebra. (a) Rotation around the $y$-axis. (b) Rotation around the $x$-axis.

\subsection{Estimation of Optimal Imaging Orientations for Stereo Fluoroscopy}

The optimal imaging orientations for stereo fluoroscopy were estimated. The accuracy estimation results are shown in Fig. 6. Panels (a) and (c) respectively depict the rotational errors of the estimation using single-plane localization accuracy as described in Section 2.2 and the error measurements obtained by using a full-search simulation. Panels (b) and (d) respectively depict the translational errors of the estimation and full-search error measurements. Similar error tendencies were observed in both sets of results. With respect to the rotation accuracy, the optimal pairs of imaging points were $\{0,60\}$ in the estimation and $\{0,75\}$ in the full-search simulation. Errors of $\{0,60\}$ in the estimation were 0.53 and 0.54 $\mathrm{mm}$, respectively, while the error of $\{0,75\}$ in the full-search simulation was 0.44 $\mathrm{mm}$. In the case of the translation accuracy, the optimal imaging points were $\{0$, $90\}$ in both results. In situations where the fluoroscopy geometry was restricted, the preoperative analysis of the optimal orientation was effective. For example, when the geometry of fluoroscopy was restricted to \pm 30 degrees, the optimal combination of imaging orientations was $\{0,30\}$ and not $\{-30,30\}$. 


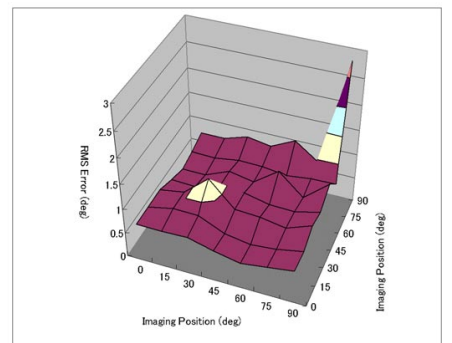

(a)

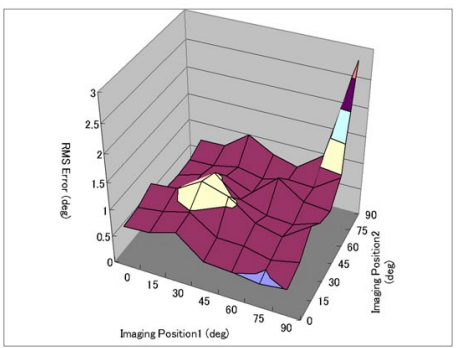

(c)

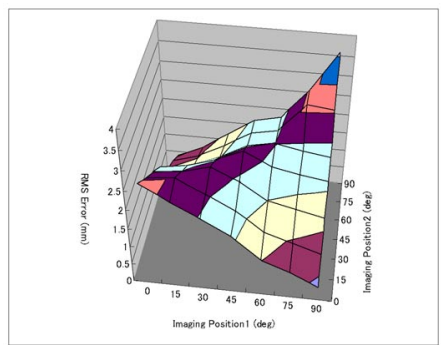

(b)

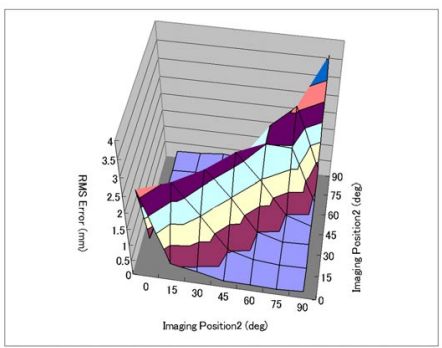

(d)

Fig. 6. Results of estimated accuracy of stereo measurement. Horizontal axes show the imaging positions of each fluoroscopy. Vertical axes show the root mean square (RMS) errors of the localized pose of the target vertebra. (a) Estimated rotation error. (b) Estimated translation error. (c) Simulated rotation error. (d) Simulated translation error.

\section{Discussion and Conclusions}

In this paper, a method of estimating the optimal imaging orientation in fluoroscopy for voxel-based $2-\mathrm{D} / 3$-D registration is proposed. The optimal imaging orientation pairs estimated experimentally were $\{0,60\}$ for rotation and $\{0,90\}$ for translation. Using optimal view estimation of stereo imaging from the registration accuracy of single-plane imaging, the system computed the optimal orientation in about 10 hours, representing a computation cost 14 times lower than that required by the full-search method. The method was validated for optimal view determination of stereo localization from the accuracy of single localization.

In this method, the stereo localization accuracy is estimated by using pseudo $\mathrm{X}$-ray images (DRRs). Although this is covenient for preoperative analysis, DRR and X-ray fluoroscopy images have different pixel intensities. Their spatial resolutions are also differ. These problems are discussed in [9]. As our next step, we intend to validate the method with respect to the limitations of its application to clinical use. The results reported here were good enough to estimate optimal imaging orientations, but were not adequate for estimating registration accuracy. 
In the future, we will take up the challenge of evaluating the effect of imaging parameters (the CT slice thickness, etc.) and integrating error components to estimate the registration accuracy and evaluate the optimal imaging parameters.

Acknowledgement: This work was partly supported by the Japan Society for the Promotion of Science (JSPS) Research for the Future Program JSPSRFTF99I00903 and the JSPS Grant-in-Aid for Scientific Research (Encouragement of Young Scientists (B) 14780281).

\section{References}

1. A. Hamadeh and P. Cinquin: "Kinematic Study of Lumber Spine Using Functional Radiographies and 3D / 2D Registration", CVRMed-MRCAS '97, pp.109-118, 1997.

2. K. Takayanagi, K. Takahashi, M. Yamagata, H. Moriya, H. Kitahara, T. Tamaki: "Using Cineradiography for Continuous Dyanmic-Motion Analysis of the Lumber Spine", SPINE, 26(17), pp. 1858-1865, 2001.

3. S. Lavallée, R. Szeliski: "Recovering the Position and Orientation of Free-Form Objects from Image Contours Using 3D Distance Map", IEEE Trans. on PAMI, 17(4), pp.378-390, 2001.

4. S.A. Banks, W.A. Hodge: "Accurate Measurement of Three-Dimensional Knee Replacement Kinematics Using Single-Plane Fluoroscopy", IEEE Trans. on Biomedical Engineering, 43(6), pp.638-649, 1996.

5. S. Zuffi, A. Leardini, F. Catani, S. Fantozzi, A. Cappello: "A Model-Based Method for Reconstruction of Total Knee Replacement Kinematics", IEEE Trans. on Medical Imaging, 18(10), 1999.

6. J. Weese, P. Penney, P. Desmedt, T.M. Buzug, D.L.G. Hill, D.J. Hawkes: "Voxel-Based 2-D/3-D Registration of Fluoroscopy Images and CT Scans for Image-Guided Surgery", IEEE Trans. on Information Technology in Biomedicine, 1(4), pp. 284-293, 1997.

7. G.P. Penney, J. Weese, J.A. Little, P. Desmedt, D.L.G. Hill, D.J. Hawkes: "A Comparison of Similarity Measures for Use in 2-D-3-D Medical Image Registration", IEEE Trans. on Medical Imaging, 17(4), pp. 586-595, 1998.

8. A. Guéziec, P. Kazanzides, B. Williamson, R.H. Taylor: "Anatomy-Based Registration of CTScan and Intraoperative X-Ray Images for Guiding a Surgical Robot", IEEE Trans. on Medical Imaging, 17(5), pp. 715-728, 1998.

9. P. Penney: "Registration of Tomographic Images to X-ray Projections for Use in Image Guided Interventions", Thesis for the degree of Doctor of Philosophy of the University of London, 1999.

10. L. Zöllei: "2D-3D Rigid-Body Registration of X-Ray Fluoroscopy and CT images", Thesis for the degree of Doctor of Philosophy of the Massachusetts Institute of Technology, 2001.

11. B. You, P. Siy, W. Anderst, and S. Tashman: "In vivo Measurement of 3-D Skeletal kinematics from Sequences of Biplane Radiographs: Application to Knee Kinematics", IEEE Trans. on Medical Imaging, 20(6), pp.514-525, 2001.

12. A.C.M. Dumay, J.H.C. Reiber, and J.J. Gerbrands: "Determination of Optimal Angiographic Viewing Angles: Basic Priciples and Evaluation Study", IEEE Trans. on Medical Imaging, 13(1), pp. 13-24, 1994.

13. Y. Sato, T. Araki, M. Hanayama, H. Naito, S. Tamura: "A Viewpoint Determination System for Stenosis Diagnosis and Quantification in Coronary Angiographic Image Acquisition", IEEE Trans. on Medical Imaging, 17(1), pp. 121-137, 1998.

14. Wilson, D.L.; Royston, D.D.; Noble, J.A.; Byrne, J.V. : "Determining X-ray Projections for Coil Treatments of Intracranial Aneurysms" IEEE Trans. on Medical Imaging, 18(10), pp. 973-980, 1999.

15. A.S. Talukdar and D.L. Wilson: "Modeling and Optimization of Rotational C-Arm Stereoscopic X-ray Angiography", IEEE Trans. on Medical Imaging 18(7), pp. 604-616, 1999.

16. M.M. Panjabi, T. Tanaka, V. Goel, D. Federico, T. Oxland, J. Duranceau, and M. Krag: "Thoracic Human Vertebrae (Quantitative Three-Dimensional Anatomy)", SPINE, 16(8), pp.888-901, 1991.

17. W. Hoff and T. Vincent: "Analysis of Head Pose Accuracy in Augmented Reality", IEEE Trans. on Visualization and Computer Graphics, 6(4), pp. 1-15, 2000. 